\title{
A Practical Method for Determining Minimum Detectable Values in Pulse-Counting Measurements
}

\author{
Yoichiro FurUKaWA, ${ }^{* \dagger}$ Manabu IwASAKI, $* *$ and Akihiro TANAKA $* * *$ \\ *DENKI KAGAKU KOGYO KABUSHIKI KAISHA, Central Research Institute, 3-5-1 Asahimachi, Machida, \\ Tokyo 194-8560, Japan \\ **Department of Computer and Information Science, Seikei University, 3-3-1 Kichijoji-Kitamachi, Musashino, \\ Tokyo 180-8633, Japan \\ ***ULVAC-PHI, Incorporated, 370 Enzo, Chigasaki, Kanagawa 253-8522, Japan
}

\begin{abstract}
The purpose of this paper is to propose a statistical method for determining minimum detectable values in a pulse-counting measurements. The output of x-ray, electron and ion-spectroscopy detectors is a series of pulses that vary in their arrival frequency according to a Poisson distribution. The analysis presented here relates this to a Normal distribution, making it consistent with the standards and methodology recommended by IUPAC and in the ISO 11843 series of international standards. The theory and limitations of doing this are presented using two types of approximations: a Simple approximation and a Square Root approximation. The variance, critical values of the response variables, capability of detection criteria and the minimum detectable values are then defined. Finally, the validity of the approximations is checked using experimental data. It is concluded that the methodology is accurate enough for practical use.
\end{abstract}

(Received September 19, 2009; Accepted December 7, 2009; Published February 10, 2010)

\section{Introduction}

Determining the minimum detectable value of signals is sometimes important in practical work. It provides a criterion for deciding when "the signal is certainly not detected", or when "the signal is significantly different to the background level". For example, it is valuable when measuring the presence of hazardous substances or surface contamination of semi-conductor materials.

There have been a number of investigations and a number of proposals concerning the methodology and the definition of the minimum detectable values in chemical analysis measurements. ${ }^{1-4}$ In 1993, representatives of the International Union of Pure and Applied Chemistry (IUPAC) and the International Organization for Standardization (ISO) met in order to reach an international consensus on the methodology, and to harmonize the different approaches. ${ }^{5-8}$ The result was the ISO 11843-1-5 standard, which uses both IUPAC recommendations and statistical testing theory in a methodology to determine the minimum detectable values. The term "minimum detectable value" replaces the more familiar "detection limit" in the ISO 11843 standards in order to bring the methodology into line with other statistical procedures.

Many types of instruments use the pulse-counting method for detecting signals. X-ray, electron and ion-spectroscopy detectors, such as X-ray diffractometers (XRD), X-ray fluorescence spectrometers (XRF), X-ray photoelectron

† To whom correspondence should be addressed.

E-mail: yoichiro-furukawa@denka.co.jp spectrometer (XPS), Auger electron spectrometer (AES), secondary ion mass spectrometers (SIMS), are of this type. Their output consists of a series of pulses produced at random, and irregular intervals that can be understood statistically using a Poisson distribution. Since the variation in a signal follows a Poisson distribution, a methodology for determining the minimum detectable values can be deduced from basic principles.

The methods used to set the minimum detectable values have for some time been in widespread use in the field of chemical analysis, although not where pulse-counting measurements are involved. The need to establish a methodology to determine the minimum detectable values in that area is recognized, and has been discussed in ISO TC201 "Surface Chemical Analysis" and by the ASTM Committee E-42 on surface chemical analysis. ${ }^{9}$

This study considers how to approximate the Poisson distribution to the Normal distribution, ensuring consistency with the IUPAC approach and, particularly, with the series of ISO $11843^{10}$ standards developed since then. Two types of approximation, called the Simple approximation and Square Root approximation, are used. Definitions are given for the variance, for critical values of the response variables, for the capability of detection criteria and for the minimum detectability levels. The two approximations are compared using experimental data, and are found to produce identical results.

Methodologies based on these two approximations are presented here. We believe they offer a practical way for analyzing the results of on-site analysis by pulse counting instrumentation. It is hoped that this paper will provide a starting point for further discussion about full integration with the IUPAC and ISO approaches. 


\section{Theory}

In general, if data are sampled from a population that has an unknown probability distribution, the distribution of the sample means will be approximately normal when the sample size, $n$, is large. This is called the central limit theorem. It is one of the most useful theorems in statistics. Although the central limit theorem still works well for small samples, particularly where the population is continuous, unimodal, and symmetrical, larger samples are needed in other situations, depending on the shape of the population. In many cases of practical interest, if $n$ is greater than 30 and the normal approximation is satisfactory regardless of the shape of the population. ${ }^{11}$

Since pulse-counting measurements are discrete, they are described by a Poisson distribution, rather than a Normal distribution. Also, the sample size, $n$, is sometimes not large enough in practical applications for a full analysis. Here, such data are processed using two types of approximation to calculate the means and the variance. These are then used to calculate the critical value of the response variable and the minimum detectable level.

The first approximation is based on the fact that, when the number of pulse events is large, the Poisson distribution tends to the Normal distribution. This type of approximation is called "the Simple approximation" here. The second approximation, called "the Square Root approximation", involves a transformation of the data so as to stabilize the variance to 1 for all measurements. This is useful when several Poisson means are to be compared, because the sample variances are independent of the Poisson means. When the mean, $\lambda$, is sufficiently large, the transformation needed to make the variance independent can be derived by approximation.

Estimation of mean value and variance by the Simple approximation

In the Poisson distribution, the probability function, $p(y, \lambda)$ is described by the following equation:

$$
p(y, \lambda)=\frac{\lambda^{y}}{y !} e^{-\lambda}
$$

where $\lambda$ is the mean value corresponding to the expected number of events in a given time; $y$ is the actual number of events recorded in that time.

The actual number of events (pulses) per unit of time will vary around the mean value. The Simple approximation works when $y>5$. In this case, the variance, $\sigma^{2}$, and the standard deviation of the distribution, $\sigma$, are given by $\sigma^{2}=\lambda$ and $\sigma=\sqrt{\lambda}$.

The Poisson random variable, $Z$, set using $E(y)=\lambda$ and $V(y)=\lambda$, is then expressed as

$$
Z=\frac{y-\lambda}{\sqrt{\lambda}}
$$

where $E(y)$ is defined as the expected value of $y$, and equals the mean, $\lambda . V(y)$ is defined as the variance of $y$, and equals the mean, $\lambda$.

Estimation of the mean value and the variance by the square root transformation approximation

In the square root transformation, the variable $y$ is converted to $Y$ using the equation $Y=2 \sqrt{y+c}$. The distribution of $Y$ has a unit variance and tends to have a Normal distribution, $N\left(2 \sqrt{\lambda+c^{\prime}}, 1\right)$, where $c$ and $c^{\prime}$ are some constants. When $c$ is

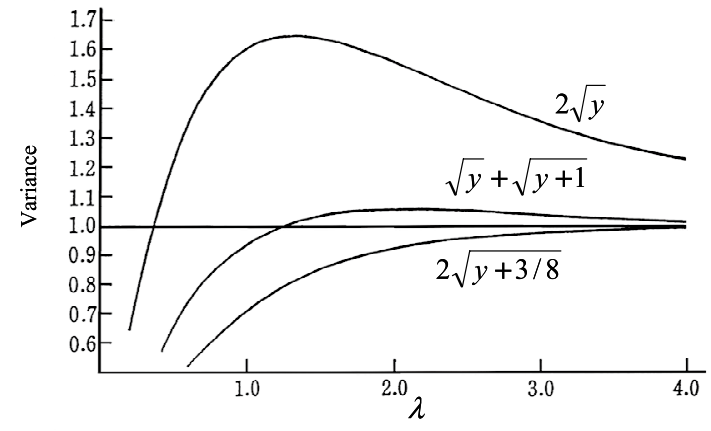

Fig. 1 Variance produced by square-root transformations. ${ }^{13}$

set to $3 / 8$, the variance of $Y$ approaches 1 more quickly than when $c$ equals 0 , and the transformed variable, $Y=2 \sqrt{y+3 / 8}$, is approximated by a Normal distribution with $N(2 \sqrt{\lambda+1 / 8}, 1) .{ }^{12-14}$ When $\lambda$ is large, the constant $c$ is less significant, and can be set to 0 . The transformation then becomes $Y=2 \sqrt{y}$, which gives a simpler equation for the Normal distribution: $N(2 \sqrt{\lambda}, 1) \cdot{ }^{14,15}$ In general, it can be shown that $V(\sqrt{y}) \approx 1 / 4$ by using the delta method. ${ }^{16}$ Then we have $V(2 \sqrt{y}) \approx 1$, which is much easier to handle in subsequent calculations. Therefore, we will use the transformation $2 \sqrt{y}$ rather than $\sqrt{y}$ here. Another transformation, $Y=\sqrt{y}+\sqrt{y+1}$, has also been suggested in the literature. ${ }^{14,17}$

The variance produced by these transformations using different values of $\lambda$ is shown in Fig. 1 .

Since only one parameter $\bar{y}$ (the mean value of the actual measurements) is used (instead of $\lambda$ ) to define a Poisson distribution, one only needs to estimate a single value. If $J$ independent measurements, $y_{1}, y_{2}, \ldots \ldots \ldots y_{\mathrm{J}}$, of the pulse count are made, the estimated mean value, $\lambda$, is expressed as

$$
\lambda=\frac{\sum_{\mathrm{i}=1}^{\mathrm{J}} y_{\mathrm{i}}}{J} .
$$

A square-root transformation of $y$ gives the transformed random variables, $2 \sqrt{y_{1}}, 2 \sqrt{y_{2}},,,,, 2 \sqrt{y_{\mathrm{J}}}$, which comply with a normal distribution, $N(2 \sqrt{\lambda}, 1)$, in which the mean value for $2 \sqrt{\lambda}$ is replaced by $\frac{1}{J} \sum_{\mathrm{i}=1}^{\mathrm{J}} 2 \sqrt{y_{\mathrm{i}}}$ when calculating the individual points.

Computation of the critical value of the response variable by the Simple approximation

The critical value, $y_{\mathrm{c}}$, is defined as the value of the response variable, $y$, when the system is considered to move from a dormant or "basic" state to an "active" state. Its value is chosen so that, when it is in the "basic" state, the system is considered to be "active" with only a small probability, $\alpha$. In other words, $y_{\mathrm{c}}$ is the lowest significant value of the measurement or signal that can be discriminated from the background noise. ${ }^{5,6,10}$

The decision about whether the signal is "detected" or "not detected" is made by comparing the arithmetic mean of the actual measurements, $\bar{y}_{\mathrm{a}}$, with the critical value, $y_{\mathrm{c}}$. The probability that $\bar{y}_{\mathrm{a}}$ exceeds $y_{\mathrm{c}}$ in the basic state should be less than or equal to an appropriate pre-selected probability, $\alpha$. A wrong decision then occurs with the probability, $\alpha$, corresponding to a signal being detected when only the background exists. This represents an "error of the first kind". 
The critical value, $y_{\mathrm{c}}$, of the response variable can generally be expressed as ${ }^{10}$

$$
P\left(\bar{y}_{\mathrm{a}}>y_{\mathrm{c}} \mid x=0\right) \leq \alpha,
$$

where $x$ refers to the state variable (often the concentration of an element or compound) to give the response variable, $y$; $\bar{y}_{\mathrm{a}}$ is the observed arithmetic mean of the actual measurements; $\alpha$ is an error of the first kind: the probability of rejecting the null hypothesis $\eta_{\mathrm{g}}=\eta_{\mathrm{b}}$, or the alternative hypothesis $\eta_{\mathrm{g}}>\eta_{\mathrm{b}}$, when the null hypothesis is true. $\eta_{\mathrm{b}}$ and $\eta_{\mathrm{g}}$ are the expected values under the actual performance conditions for the responses of the basic state, $x_{0}$ and a sample with the state variable equal to $x_{\mathrm{g}}$. The probability is $\alpha$ of detecting (erroneously) that a system is not in the basic state when it is in the basic state. Equation (4) gives the probability that $\bar{y}_{\mathrm{a}}>y_{\mathrm{c}}$ under the condition that $x=0$. If $y$ is large enough and its deviation tends to the Poisson deviation, $\sigma_{0}$ equals $\sqrt{y}$, and the critical value of the response variable is given by the following simplified version of Eq. (4):

$$
y_{\mathrm{c}}=\bar{y}_{\mathrm{b}} \pm Z_{1-\alpha} \sigma_{0} \sqrt{\frac{1}{J}+\frac{1}{K}} .
$$

Also, if $\sigma_{0}$ is replaced by $\sqrt{\bar{y}_{\mathrm{b}}}$, and then

$$
y_{\mathrm{c}}=\bar{y}_{\mathrm{b}} \pm Z_{1-\alpha} \sqrt{\bar{y}_{\mathrm{b}}} \sqrt{\frac{1}{J}+\frac{1}{K}}
$$

where $y_{\mathrm{c}}$ is the critical value of the response variable; $Z_{1-\alpha}$ represents the $(1-\alpha)$-quantile of the standard normal variable; $\sigma_{0}$ is the standard deviation of the signal under the null hypothesis (true value for $x=0$ ); $\bar{y}_{\mathrm{b}}$ is the observed arithmetic mean of the measurements of the basic state with state variable $x_{0} ; J$ is the number of replications of measurements on the basic state; and $K$ is the number of replications of measurements on the actual state.

Note that the + sign is used when the response variable increases in line with the state variable and the - sign is used when the response variable decreases in line with the state variable. In general, when the value of the state variable in the basic state (the baseline for the response variable) is known without a significant error, then $\sigma_{0}=\sigma_{\mathrm{b}}$. The latter is estimated through $\bar{y}_{\mathrm{b}}$, the standard deviation of the repeated measurements of the response variable in the basic state. As mentioned in the Simple approximation section, $\sqrt{\bar{y}_{\mathrm{b}}}$ gives an estimate of $\sigma_{\mathrm{b}}$.

The concept of the critical value is illustrated in Fig. $2{ }^{10}$

Sufficient capability of the detection criterion by a Simple approximation

The sufficient capability of detection criterion enables decisions to be made about the detection of a signal by comparing the critical value probability with a specified value, $1-\beta$. If the criterion is satisfied, it may be concluded that the minimum detectable value, $x_{\mathrm{d}}$, is less than or equal to the value of the state variable, $x_{\mathrm{g}}$. The minimum detectable value then defines the smallest expectation of the response variable, $\eta_{\mathrm{g}}$, for which an incorrect decision occurs with a probability $\beta$ that there is no signal, only background. This is termed an "error of the second kind". ${ }^{10}$

If the standard deviation of the responses for a given value $x_{\mathrm{g}}$ is $\sigma_{\mathrm{g}}$, the criterion for the probability to be greater than or equal to $1-\beta$ is defined by Eq. (7), and is actually given by Eqs. (8) and (9):

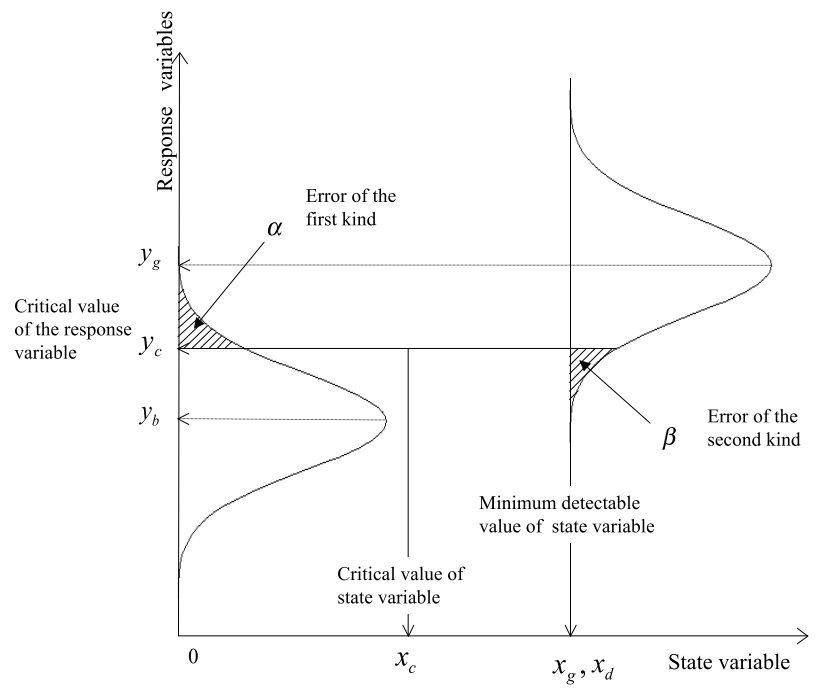

Fig. 2 Relation between the critical value of the response variable and the minimum detectable value of the state variable. ${ }^{10}$

$$
\eta_{\mathrm{g}} \geq y_{\mathrm{c}}+Z_{1-\beta} \sqrt{\frac{1}{J} \sigma_{\mathrm{b}}^{2}+\frac{1}{K} \sigma_{\mathrm{g}}^{2}}
$$

If $y_{\mathrm{c}}$ is replaced by $\eta_{\mathrm{b}}+Z_{1-\alpha} \sigma_{\mathrm{b}} \sqrt{\frac{1}{J}+\frac{1}{K}}$, defined in Eq. (5), then

$$
\eta_{\mathrm{g}}-\eta_{\mathrm{b}} \geq Z_{1-\alpha} \sigma_{\mathrm{b}} \sqrt{\frac{1}{J}+\frac{1}{K}}+Z_{1-\beta} \sqrt{\frac{1}{J} \sigma_{\mathrm{b}}^{2}+\frac{1}{K} \sigma_{\mathrm{g}}^{2}},
$$

where $1-\alpha$ and $1-\beta$ are the confidence levels.

With $\beta=\alpha, K=J$ and under the assumption that $\sigma_{\mathrm{g}} \geq \sigma_{\mathrm{b}}$ (it is unusual for the standard deviation to decrease as the state variable increases), the criterion simplifies to

$$
\frac{\eta_{\mathrm{g}}-\eta_{\mathrm{b}}}{\sqrt{\sigma_{\mathrm{b}}^{2}+\sigma_{\mathrm{g}}^{2}}} \geq \frac{2 Z_{1-\alpha}}{\sqrt{J}}
$$

The relation between the critical value of the response variable and the minimum detectable value of state variable is shown in Fig. $2 .{ }^{10}$

\section{Confirmation of the sufficient capability of detection criterion by a Simple approximation}

The standard deviations and expected values of the responses are usually unknown, so an assessment using criterion Eq. (9) has to be made of the experimental data. The expression on the left-hand side of the simplified criterion Eq. (9) is unknown, whereas that on the right-hand side is known.

From a validation experiment with $N$ measurements of the basic state and $N$ measurements of a sample with the state variable equal to $x_{\mathrm{g}}$, one can obtain $\eta_{\mathrm{g}}-\eta_{\mathrm{b}}$ on the left hand side of inequality Eq. (9). The difference in the means is then represented by:

$$
\begin{aligned}
\left(\bar{y}_{\mathrm{g}}-\bar{y}_{\mathrm{b}}\right)-Z_{1-\alpha} \sqrt{\frac{1}{J} \sigma_{\mathrm{b}}^{2}+\frac{1}{K} \sigma_{\mathrm{g}}^{2}} & \leq\left(\eta_{\mathrm{g}}-\eta_{\mathrm{b}}\right) \\
& \leq\left(\bar{y}_{\mathrm{g}}-\bar{y}_{\mathrm{b}}\right)+Z_{1-\alpha} \sqrt{\frac{1}{J} \sigma_{\mathrm{b}}^{2}+\frac{1}{K} \sigma_{\mathrm{g}}^{2}} .
\end{aligned}
$$

With $\beta=\alpha, K=J$; also, under the assumption that $\sigma_{\mathrm{g}} \geq \sigma_{\mathrm{b}}$, Eq. (10) is rearranged as 


$$
\frac{\bar{y}_{\mathrm{g}}-\bar{y}_{\mathrm{b}}}{\sqrt{\sigma_{\mathrm{b}}^{2}+\sigma_{\mathrm{g}}^{2}}}-Z_{1-\alpha} \sqrt{\frac{1}{J}} \leq \frac{\eta_{\mathrm{g}}-\eta_{\mathrm{b}}}{\sqrt{\sigma_{\mathrm{b}}^{2}+\sigma_{\mathrm{g}}^{2}}} \leq \frac{\bar{y}_{\mathrm{g}}-\bar{y}_{\mathrm{b}}}{\sqrt{\sigma_{\mathrm{b}}^{2}+\sigma_{\mathrm{g}}^{2}}}+Z_{1-\alpha} \sqrt{\frac{1}{J}}
$$

The smaller expression on the left-hand side of Eq. (11) is chosen and compared to the right-hand side of Eq. (9), giving

$$
\frac{\eta_{\mathrm{g}}-\eta_{\mathrm{b}}}{\sqrt{\sigma_{\mathrm{b}}^{2}+\sigma_{\mathrm{g}}^{2}}}=\frac{\bar{y}_{\mathrm{g}}-\bar{y}_{\mathrm{b}}}{\sqrt{\sigma_{\mathrm{b}}^{2}+\sigma_{\mathrm{g}}^{2}}}-Z_{1-\alpha} \sqrt{\frac{1}{J}} \geq \frac{2 Z_{1-\alpha}}{\sqrt{J}} .
$$

An approximate $100(1-\alpha) \%$ lower confidence limit (CL) for $\left(\eta_{\mathrm{b}}-\eta_{\mathrm{g}}\right) / \sqrt{\sigma_{\mathrm{b}}^{2}+\sigma_{\mathrm{g}}^{2}}$ is given by

$$
\mathrm{CL}=\frac{\bar{y}_{\mathrm{g}}-\bar{y}_{\mathrm{b}}}{\sqrt{\bar{y}_{\mathrm{g}}+\bar{y}_{\mathrm{b}}}}-\frac{Z_{1-\alpha}}{\sqrt{N}}
$$

where $\bar{y}_{\mathrm{g}}$ is the observed arithmetic mean for the sample with the state variable equal to $x_{\mathrm{g}} ; N$ is the number of replications of measurements in assessment of the capability of detection.

If the lower confidence limit of $\left(\eta_{\mathrm{b}}-\eta_{\mathrm{g}}\right) / \sqrt{\sigma_{\mathrm{b}}^{2}+\sigma_{\mathrm{g}}^{2}}$ satisfies criterion Eq. (9), it is concluded that the minimum detectable value, $x_{\mathrm{d}}$, is less than or equal to $x_{\mathrm{g}}$. Note that, for relatively large values of $N$, the lower confidence limit Eq. (13) will suffice.

Computation of the critical value of the response variable by the square-root transformation approximation

If $2 \sqrt{y_{\mathrm{i}}}$ is normally distributed with a standard deviation of $\sigma_{0}$, then the critical value of the response variable, $y_{\mathrm{c}}$, is given by the following simplified version of Eq. (5):

$$
\begin{aligned}
2 \sqrt{y_{\mathrm{c}}}=2 \sqrt{\bar{y}_{\mathrm{b}}} \pm Z_{1-\alpha} \sigma_{0} \sqrt{\frac{1}{J}+\frac{1}{K}}=2 \sqrt{\bar{y}_{\mathrm{b}}} \pm Z_{1-\alpha} \sqrt{\frac{1}{J}+\frac{1}{K}} \\
\\
\left(\text { since } \sigma_{0}^{2} \cong 1, \sigma_{0} \cong 1\right) .
\end{aligned}
$$

Note that the + sign is used when the response variable increases in line with the state variable, and the - sign is used when the response variable decreases in line with the state variable.

Sufficient capability of detection criterion by the square-root transformation

If the standard deviation of the response for a given value $x_{\mathrm{g}}$ of the state variable is $\sigma_{\mathrm{g}}$, the criterion for the probability to be greater than or equal to $1-\beta$ is given by:

$$
2 \sqrt{\eta_{\mathrm{g}}} \geq 2 \sqrt{y_{\mathrm{c}}}+Z_{1-\beta} \sqrt{\frac{1}{J} \sigma_{\mathrm{b}}^{2}+\frac{1}{K} \sigma_{\mathrm{g}}^{2}} .
$$

If $2 \sqrt{y_{\mathrm{c}}}$ is replaced by $2 \sqrt{\eta_{\mathrm{b}}}+Z_{1-\alpha} \sigma_{\mathrm{b}} \sqrt{\frac{1}{J}+\frac{1}{K}}$ defined in Eq. (5), then

$$
2 \sqrt{\eta_{\mathrm{g}}}-2 \sqrt{\eta_{\mathrm{b}}} \geq Z_{1-\alpha} \sigma_{\mathrm{b}} \sqrt{\frac{1}{J}+\frac{1}{K}}+Z_{1-\beta} \sqrt{\frac{1}{J} \sigma_{\mathrm{b}}^{2}+\frac{1}{K} \sigma_{\mathrm{g}}^{2}} .
$$

With $\beta=\alpha, K=J$, and under the assumption that $\sigma_{\mathrm{g}} \geq \sigma_{\mathrm{b}}$, the criterion simplifies to

$$
\sqrt{\eta_{\mathrm{g}}}-\sqrt{\eta_{\mathrm{b}}} \geq \sqrt{2} Z_{1-\alpha} \sqrt{\frac{1}{J}}
$$

Confirmation of the sufficient capability of detection criterion by the square-root transformation

An assessment using criterion Eq. (17) has to be made of the experimental data in the same way as for the Simple approximation.
Using a validation experiment with $N$ measurements of both the basic state and a sample state variable, one gets a range for the interval on the left-hand side of criterion Eq. (17) within which $Z_{1-\alpha}$ and $\alpha$ can be expected to lie with a pre-specified probability, $1-\alpha$.

$$
\begin{aligned}
\left(2 \sqrt{\bar{y}_{\mathrm{g}}}-2 \sqrt{\bar{y}_{\mathrm{b}}}\right)- & Z_{1-\alpha} \sqrt{\frac{1}{J} \sigma_{\mathrm{b}}^{2}+\frac{1}{K} \sigma_{\mathrm{g}}^{2}} \leq\left(2 \sqrt{\eta_{\mathrm{g}}}-2 \sqrt{\eta_{\mathrm{b}}}\right) \\
\leq & \left(2 \sqrt{\bar{y}_{\mathrm{g}}}-2 \sqrt{\bar{y}_{\mathrm{b}}}\right)+Z_{1-\alpha} \sqrt{\frac{1}{J} \sigma_{\mathrm{b}}^{2}+\frac{1}{K} \sigma_{\mathrm{g}}^{2}}
\end{aligned}
$$

With $\beta=\alpha, K=J$, and under the assumptions that $\sigma_{\mathrm{g}} \geq \sigma_{\mathrm{b}}$ and the variance is stabilized to $1\left(\sigma_{0}^{2} \cong 1\right.$ in the square root transformation); also, the bound of interval estimate becomes

$$
\begin{aligned}
&\left(2 \sqrt{\bar{y}_{\mathrm{g}}}-2 \sqrt{\bar{y}_{\mathrm{b}}}\right)-Z_{1-\alpha} \sqrt{\frac{1}{J}(1+1)} \leq\left(2 \sqrt{\eta_{\mathrm{g}}}-2 \sqrt{\eta_{\mathrm{b}}}\right) \\
& \leq\left(2 \sqrt{\bar{y}_{\mathrm{g}}}-2 \sqrt{\bar{y}_{\mathrm{b}}}\right)+Z_{1-\alpha} \sqrt{\frac{1}{J}(1+1) .}
\end{aligned}
$$

The smaller expression on the left-hand side of Eq. (19) is chosen and compared to the constant criterion value of Eq. (17) as follows:

$$
\sqrt{\eta_{\mathrm{g}}}-\sqrt{\eta_{\mathrm{b}}}=\left(\sqrt{\bar{y}_{\mathrm{g}}}-\sqrt{\bar{y}_{\mathrm{b}}}\right)-Z_{1-\alpha} \sqrt{\frac{1}{2 J}} \geq \sqrt{2} Z_{1-\alpha} \sqrt{\frac{1}{J}} .
$$

An approximate $100(1-\alpha) \%$ lower confidence limit (CL) for $\sqrt{\eta_{\mathrm{g}}}-\sqrt{\eta_{\mathrm{b}}}$ is given by

$$
\mathrm{CL}=\left(\sqrt{\bar{y}_{\mathrm{g}}}-\sqrt{\bar{y}_{\mathrm{b}}}\right)-Z_{1-\alpha} \sqrt{\frac{1}{2 N}} .
$$

If the lower confidence limit for $\sqrt{\eta_{\mathrm{g}}}-\sqrt{\eta_{\mathrm{b}}}$ satisfies criterion Eq. (17), one concludes that the minimum detectable value, $x_{\mathrm{d}}$, is less than or equal to $x_{\mathrm{g}}$. For relatively large values of $N$, the lower confidence limit may be considered to be sufficient.

\section{Required Experimental Conditions}

In practice, the conditions under which a pulse counting measurement is made are usually specified by the experimental set-up. The number of pulses that are detected increases with both the time and with the width of the region over which the spectrum is being observed. These two parameters should therefore be noted and not changed during the course of the measurement.

The following conditions are essential for determining the minimum detectable value using the analysis presented here:

(1) Both the signal and the background should have Poisson distributions.

(2) The spectra under consideration should not have received any data treatment, such as smoothing and derivation.

(3) Time interval: A single measurement over a longer period of time is preferable to several shorter measurements. A measurement taken for over $1 \mathrm{~s}$ is better than 10 measurements over $100 \mathrm{~ms}$ each. The approximation of the Poisson distribution by the Normal distribution is more reliable with higher mean values.

(4) The number of measurements: Since only mean values are used in the approximations presented here, repeated measurements are needed to determine them. Moreover, the detectability increases with the number of measurements, as shown in equalities Eqs. (9), (13), (17) and (21). 


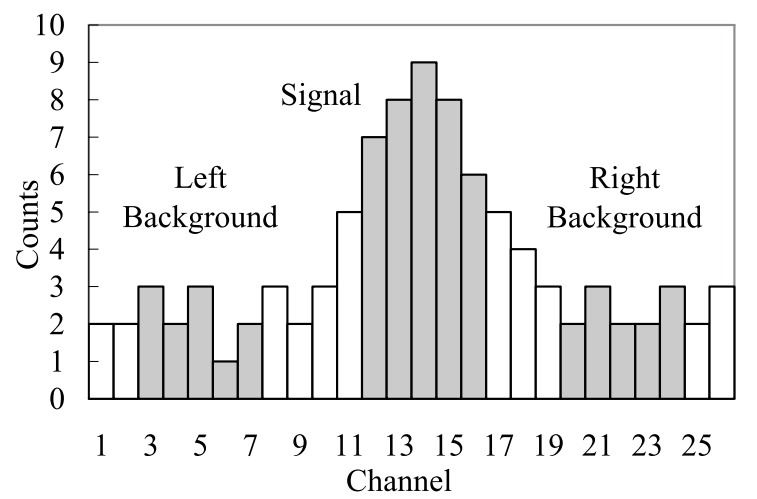

Fig. 3 Specification of signal regions.

(5) Selecting the number of channels used by the detector: There should be no overlap of neighboring peaks. The number of channels used to measure the background and sample spectra should be identical. See Fig. 3 .

(6) Peak width: The full width at half maximum (FWHM) is the recommended coverage for monitoring a single peak, and is preferable to measurements based on the top and/or bottom of the peak with noise. The appropriate FWHM should be determined beforehand by measurements on a standard sample. An identical value of the FWHM width should be adopted for both background and sample measurements.

For the same reason, the equivalent number of channels should be identical when measuring the peak and background signals to determine the minimum detectable value.

\section{Results and Discussion}

Application limits - the accuracy of the approximations

The judgment criteria set specific statistical values for assessing the detection capability of pulse count measurements, and whether a particular signal is significant or not. They are only applicable when variations in both the background and the signal are describable by the Poisson distribution. Users need to convince themselves beforehand that this is the case.

The number of counts is similarly important. The Central Limit theorem indicates that the Normal approximation to the Poisson distribution only works well when the number of counts is large. The Simple approximation is based on this. Figures 4(a) and 4(b) show the difference between a Poisson distribution and corresponding Normal distribution when $\lambda$, the Poisson mean corresponding to the expected number of events observed in a given time interval, is equal to 10 and 100, respectively. For a small $\lambda$, such as 10, a small difference between the two distributions can be observed; for a large $\lambda$, such as 100 , the two distributions are almost the same.

The square root transformation is slightly different. Its purpose is to make the variance independent of the Poisson mean, $\lambda$. When several mean values are compared, as in analysis of the variance, it is easier if the variances of the sampling distributions are the same.

Such criteria are most important for high-precision analysis. The best measurement conditions should be used to give the highest number of repeated measurements and the highest number of counts.
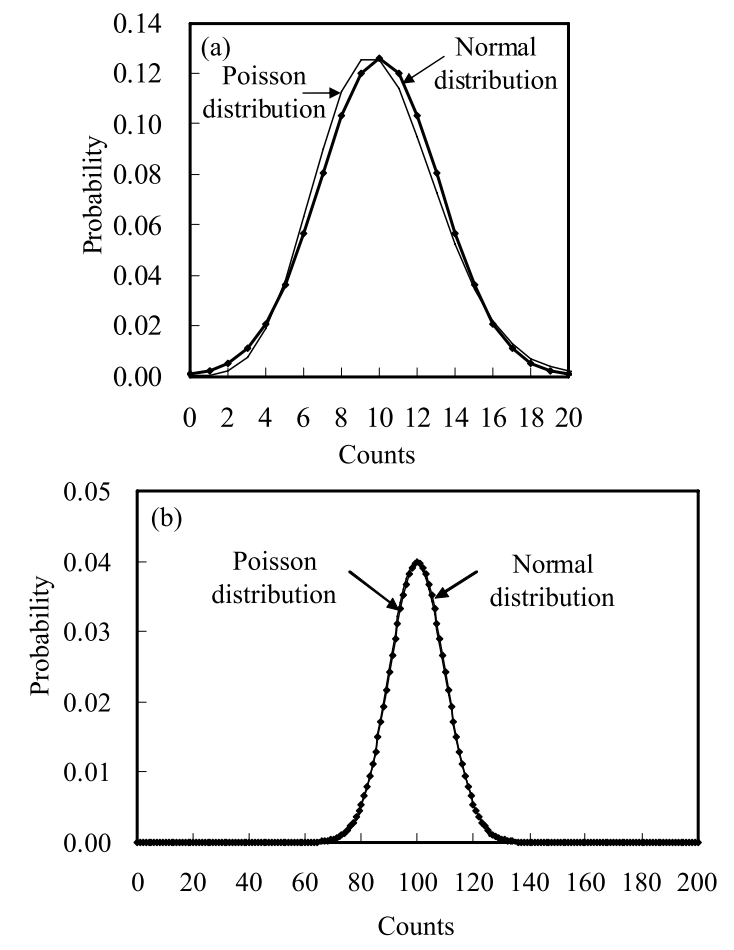

Fig. 4 Difference between the Poisson distribution and Normal distribution. (a) The probability distribution when $\lambda$ equals to 10 ; (b) when $\lambda$ equals to 100 .

Confirmation of proposed methodology—the minimum detectability

In computing the minimum detectable values, the number of repetitions, $J$ and $K$, is set to 1 in order to obtain the highest reliability. For the $95 \%$ lower confidence limit, $\alpha=\beta=0.05$, reference tables on the cumulative standard Normal distribution show the $1-\alpha$ quantile of the standard normal variable, $Z_{1-\alpha}$, to be 1.645. The right-hand side of Eq. (9) then equates to 3.29 in the Simple approximation.

In the inequality equation $\frac{\eta_{\mathrm{g}}-\eta_{\mathrm{b}}}{\sqrt{\sigma_{\mathrm{g}}^{2}+\sigma_{\mathrm{b}}^{2}}} \geq \frac{2 Z_{1-\alpha}}{\sqrt{J}}, \frac{2 Z_{1-\alpha}}{\sqrt{J}}$ equals 3.29.

In the same way, the right-hand side of Eq. (17) is 2.326 using the Square Root transformation.

There, in the inequality equation $\sqrt{\eta_{\mathrm{g}}}-\sqrt{\eta_{\mathrm{b}}} \geq \sqrt{2} Z_{1-\alpha} \sqrt{\frac{1}{J}}$, $\sqrt{2} Z_{1-\alpha} \sqrt{\frac{1}{J}}$ equals 2.326 .

The minimum detectable values, $\bar{y}_{\mathrm{g}}$ are calculated to satisfy the sets of Eqs. (9) and (13), (17) and (21), respectively. The absolute minimum detectable values can also be computed. These values are obtained when infinity is used for $N$, the number of repeated measurements.

The computed $\bar{y}_{\mathrm{g}}$ and the absolute minimum detectable values are listed in Table 1. The agreement is exact, even though the two approximations have different theoretical backgrounds and have been calculated independently.

\section{Comparison of proposed methodology with experimental data}

Organic contamination on the surface of a silicon wafer was detected using the carbon 1s X-ray photoelectron spectroscopy (XPS) spectrum. The spectrum of measurement for the blank concentration, $x_{\mathrm{b}}$, and the contaminant concentration, $x_{\mathrm{g}}$, was 
Table 1 The minimum detectable values

\begin{tabular}{crccc}
\hline$N$ & $\bar{y}_{\mathrm{b}}$ & $\begin{array}{c}\text { Type of } \\
\text { approximation }\end{array}$ & $\bar{y}_{\mathrm{g}}$ & $\begin{array}{c}\text { Absolute minimum } \\
\text { detectable value }\end{array}$ \\
\hline \multirow{2}{*}{10} & 60 & Simple & 109 & 101 \\
& 750 & Simple & 905 & 883 \\
10 & 60 & Square root & 109 & 101 \\
& 750 & Square root & 905 & 883 \\
\hline
\end{tabular}

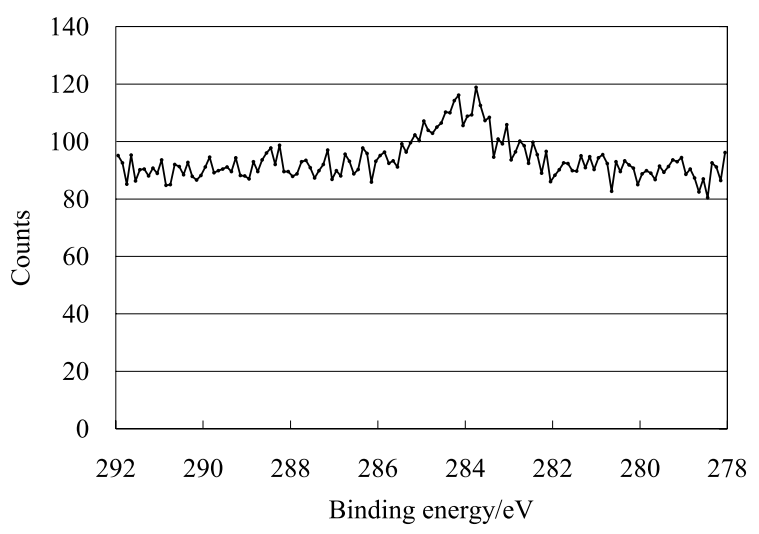

Fig. 5 XPS carbon 1s spectrum.

given in Fig. 5. The capability of the detection calculation used $J=K=1$ and $\alpha=\beta=0.05$.

The measurements conditions were as follows: instrument used, Quantera SXM (Ulvac-PHI Inc.); X-ray source, Al K monochromatic X-ray; X-ray source output power, $0.9 \mathrm{~W}$; channel width, $0.10 \mathrm{eV}$; number of channels, 14 (totaling $1.3 \mathrm{eV}$, and giving FWHM coverage of binding energy for both the background $288.65-287.35 \mathrm{eV}$ and peak areas 284.55 - $283.25 \mathrm{eV}$; number of measurements, 7; accumulation time for each channel, $100 \mathrm{~ms}$.

Analysis by the Simple approximation

The experiment gave counts for $\bar{y}_{\mathrm{b}}, \bar{y}_{\mathrm{g}}$ of 1288 and 1523 , respectively. Using these values, a $95 \%$ lower confidence limit of $\frac{\bar{y}_{\mathrm{g}}-\bar{y}_{\mathrm{b}}}{\sqrt{\bar{y}_{\mathrm{g}}+\bar{y}_{\mathrm{b}}}}-\frac{Z_{1-\alpha}}{\sqrt{N}}$, calculated according to Eq. (13) gave $\frac{1523-1288}{\sqrt{1523+1288}}-\frac{1.645}{\sqrt{7}}=3.811$.

This is greater than $2 Z_{1-\alpha} / \sqrt{J}=3.29$ in Eq. (9). We can therefore conclude, with better than $95 \%$ confidence, that the surface of a silicon wafer is contaminated with hydrocarbon.

Analysis by the square root transformation approximation A $95 \%$ lower confidence limit of $\left(\sqrt{\bar{y}_{\mathrm{g}}}-\sqrt{\overline{y_{\mathrm{b}}}}\right)-Z_{1-\alpha} \sqrt{\frac{1}{2 N}}$, calculated according to Eq. (21) gave $(\sqrt{1523}-\sqrt{1288})-$ $1.645 / \sqrt{2 * 7}=2.697$. This is greater than $\sqrt{2} Z_{1-\alpha} \sqrt{1 / J}=2.326$ in Eq. (17). Again, we can conclude, with better than $95 \%$ confidence, that the surface of silicon wafer is contaminated with hydrocarbon. Both methods therefore agree that, in this case, the signal was real and that the surface was contaminated.

\section{Conclusion}

In the present study, statistical theory has been used to analyze pulse-counting measurements and to decide whether a specific signal is significant or not. Two approximation methods were examined. Both conform to the IUPAC and ISO harmonized concepts, as given in the series of ISO 11843 standards. Statistical formulae were applied to experimental data to verify the proposed methods. The results were found to be consistent and almost identical.

When using the proposed methods, there are some limitations. A difference between Poisson and Normal distributions is expected when the number of counts is small. The minimum detectable values under the two approximations depend on both the number of counts observed and the number of repeat measurements, even though the way they approximate the Normal distribution is different. When high-precision is needed, it is important to select optimal measurement conditions in order to allow a high number of repetitions and a high number of counts.

This study proposes two ways of determining the minimum detectable values. It does not favor one over the other, but shows that there is a small difference, especially when the number of counts is small. It is suggested that the results are analyzed using both methods and then compared. They should be the same. If not, the worst result should be used if hazardous substances are involved.

The issue of background subtraction is not considered in this study, since it is not essential for the analysis. In practice, difficulties can arise when the background is sloping or irregular. As a minimum, systematic noise should be subtracted. The practicality of doing this will be discussed in a future publication.

We conclude that the proposed methodology can be recommended as satisfying the needs of on-site analysis by pulse-counting instrumentation.

\section{References}

1. H. Kaiser, Spectrochim. Acta, 1947, 3, 40.

2. L. A. Currie and G. Svehla, Anal. Chem., 1968, 40, 586.

3. ACS Committee on Environmental Improvement, Anal. Chem., 1980, 52, 2242.

4. L. A. Currie and J. Res. NBS, 1985, 90, 409.

5. L. A. Currie and G. Svehla, Pure Appl. Chem., 1994, 66, 595.

6. L. A. Currie, Pure Appl. Chem., 1995, 67, 1699.

7. L. A. Currie, Chemom. Intell. Lab. Syst., 1997, 37, 151.

8. ISO, "Guide to the Expression of Uncertainty in Measurement", 1995, ISBN 92-67-10188-9.

9. D. R. Baer, Surf. Interface Anal., 2007, 39, 283.

10. ISO TC ISO 11843-1 Capability of detection-Part 1: Terms and definitions (1997), ISO 11843-2 Capability of detection-Part 1: Methodology in the non linear calibration case (2000), ISO 11843-3 Capability of detection-Part 3: Methodology for the determination of the critical value for the response variable when no calibration data are used (2003), ISO 11843-4 Capability of detection-Part 4: Methodology for comparing the minimum detectable value with a given value (2003), ISO 11843-5 Capability of detection-Part 5: Methodology in the non linear calibration case (2008).

11. D. C. Montgomery and G. C. Runger, "Applied Statistics and Probability for Engineers", 2006, John Wiley and 
Sons, Inc.

12. F. J. Anscomber, Biometrika, 1948, 35, 246.

13. K. Takeuchi and K. Fujino, "Binominal Distribution and Poisson Distribution", 1981, Tokyo University Shuppan Kai.

14. N. L. Johnson and S. Kotz, "Discrete Distributions", 1969,
Houghton Mifflin Company, Boston.

15. M. S. Bartlett, Journal of the Royal Statistical Society, 1936, 3(Suppl.), 68.

16. G. W. Oehlert, The American Statistician, 1992, 46, 27.

17. M. F. Freeman and J. W. Tukey, Annals of Mathematical Statistics, 1950, 21, 607. 